\title{
Quadrotor trajectory tracking by using fixed-time differentiator
}

\author{
Bai-Hui Du ${ }^{\mathrm{a}}$, Andrey Polyakov ${ }^{\mathrm{b}}$, Gang Zheng ${ }^{\mathrm{b}}$ and Quan Quan ${ }^{\mathrm{a}}$

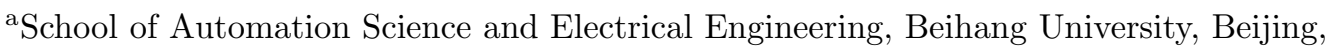 \\ China; ${ }^{b}$ Non-A team, Inria Lille-Nord Europe, Villeneuve d'Ascq, France
}

\author{
ARTICLE HISTORY \\ Compiled December 19, 2017
}

\begin{abstract}
This paper proposes a fixed-time differentiator running in parallel with a feedback linearization-based controller, which allows quadrotors to track a given trajectory. The fixed-time differentiator estimates outputs' derivatives in a predefined convergence time, largely compensating for initial condition problems and solving the delay problem. Combined with a state reconstruction step, a whole observer-estimatorcontroller scheme for trajectory tracking problem of quadrotors can be constructed. Also, an LMI optimization-based algorithm to tune parameters of the differentiator is also developed here. The high performance of the proposed model is illustrated by simulation results.
\end{abstract}

\section{KEYWORDS}

Fixed-time differentiator, LMI optimization, Feedback linearization, Trajectory tracking.

\section{Introduction}

Compared with fixed-wing aircrafts and helicopters, quadrotors are easier to use in the case of specific tasks with good performance and a high level of autonomy, see Austin (2010) and Quan (2017). A quadrotor is a nonlinear under-actuated dynamic system with four control inputs and six degrees of freedom, as is explained in Mahony, Kumar, and Corke (2012), Balas (2007), and Quan (2017). Therefore, the control problem of trajectory tracking for a quadrotor is highly demanding not only for the nonlinearity but also for the stability, the robustness, and dynamic properties.

In order to guarantee the agility and the controllability of quadrotors, the flight control system should be able to track given trajectories with high accuracy. Numerous kinds of research have been conducted to study tracking control problem of quadrotors. Bouabdallah (2006) applied some generally used control methods to quadrotors, such as the PID technique, the LQR control method, etc. Then, Bouabdallah and Siegwart (2007) proposed a combination of PID and back-stepping approach Then a combination of has been proposed for attitude, altitude and position control respectively, aiming to solve tracking control problem of quadrotors. In the paper of Adigbli, Grand, Mouret, and Doncieux (2007), three control approaches - back-stepping controller, sliding mode controller, and feedback controller - were designed for quadrotor to track set-points. Also, their performances were compared. A discrete PID controller 
for quadrotors was developed by Khan and Kadri (2014), permitting the quadrotor to move in space. And the results have been validated by a hardware-in-loop simulation.

Most of these studies employ a hierarchical control scheme consisting of attitude control, altitude control and position control to realize an autonomous trajectory tracking, as is shown in Fig.1. Since the quadrotor dynamic system is nonlinear and underactuated, the central issue of the trajectory tracking control problem is the decoupling problem.

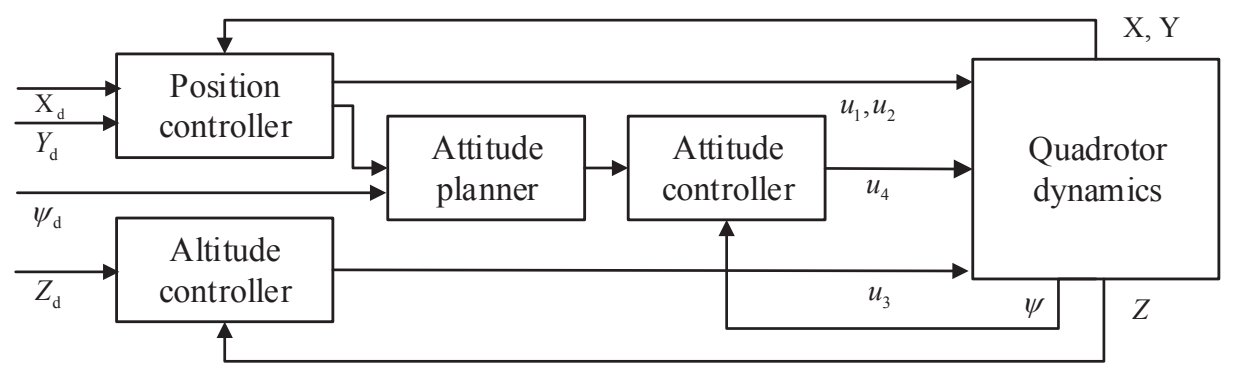

Figure 1. A hierarchical control scheme for autonomous trajectory tracking

However, the hierarchical control scheme is based on an approximate linear model, which decouples the control problem by approximation around given set-points. This approximation results in limits for the pitch and roll angles of quadrotors. To avoid problems caused by the small-angle approximation, researchers proposed numerous advanced control methods, such as the feedback linearization method mentioned in the book of Isidori (1989), the fixed-time stabilization studied by Polyakov (2012), etc. The feedback linearization method is an exact linearization from the point of view of global input-output linearization, see Nijmeijer and van der Schaft (1990). The feedback linearization-based controller can render the quadrotor dynamic system linear and controllable. Moreover, in the paper of Mistler, Benallegue, and M'Sirdi (2001), simulations were also carried out to confirm the stability and the robustness of the vehicle in the presence of environmental disturbances and parametric uncertainties. However, this approach requires full information of the system states, including the third derivatives of the output signals. Thus an efficient differentiator design for the output signals becomes indispensable.

The real-time differentiation has always been an interesting and highly demanding problem, regarding the combination between robustness and exactness with respect to noises and measurement errors. Various kinds of research have been conducted to design a robust exact differentiator for both linear and nonlinear systems. In the paper of Cruz-Zavala, Moreno, and Fridman (2010), a super-twisting algorithm-based uniform robust exact differentiator was studied, which provides exact derivatives of the input in a finite convergence time. Another commonly used approach is the high order sliding mode differentiator (HOSM), see Levant (1998), etc. The high order sliding mode differentiator is a classical approach for its insensitivity to unknown inputs and its finite-time convergence ${ }^{1}$ property, as in Levant (2003). The convergence time of

\footnotetext{
${ }^{1} \mathrm{~A}$ system is called finite-time stable means that the system reaches in the steady state and remains there in
} 
this type of differentiators varies with different initial conditions. Slow response times, however, may lead to severe problems in practical applications. Therefore, more and more researchers began to focus on fixed-time differentiators ${ }^{2}$, of which the convergence time is bounded by a fixed value independent of initial conditions, see Polyakov, Efimov, and Perruquetti (2015a). Moreover, hybrid fixed-time differentiators have also become a focus recently. Angulo, Moreno, and Fridman (2013) propose an arbitraryorder differentiator which provides the uniform convergence property within a finite settling time. This kind of differentiator design guarantees the exactness of the derivatives' estimation in a finite time and the independence to various initial differentiation errors. Similarly, in the paper of Rio and Teel (2016), a hybrid fixed-time observer for single output linear system was studied, which also combines the exactness property after a fixed time and the uniform convergence property.

As the feedback linearization approach transforms the nonlinear quadrotor dynamic system into a linear form where the controller requires the third derivatives of the system states, it is instrumental to introduce a real-time differentiator as an observer and an estimator in the control loop. A high order sliding mode differentiator is an appropriate approach for its insensitivity to disturbances and finite-time transient. In the paper of Benallegue, Mokhtari, and Fridman (2007), a high-order sliding mode observer was designed for quadrotors which provides satisfying control performance in the case of external disturbances and parametric uncertainties. To guarantee a faster response time with respect to significant deviations from equilibrium, a fixed-time differentiator is more suitable for practical applications, such as the quadrotor dynamics. A primary difficulty of the fixed-time differentiator applications is the parameter tuning problem, which is directly related to the fixed settling time. In the paper of Basin, Yu, and Shtessel (2016), non-recursive higher order sliding mode differentiators with finite and fixed convergence time were studied. The settling time, however, is implicit and the time estimation is slightly complicated.

The main contribution of this paper is to apply a fixed-time differentiator for quadrotor model to estimate outputs' derivatives, running in parallel with a dynamic feedback linearization-based controller. The convergence time of differentiators is bounded by a fixed value independent of the initial differentiation error. Furthermore, using an LMI optimization-based parameter tuning algorithm, the gain matrix of differentiators as well as the convergence time can be quickly settled. A control strategy comparison to the commonly used PID technique has been given to illustrate the performance of the feedback linearization-based controller. Numerical simulations have been conducted at the end to present the computation of the whole observer-estimatorcontroller model and the effectiveness of the proposed scheme for trajectory tracking problems of quadrotors.

The remainder of this paper is organized as follows: Section 2 introduces the quadrotor dynamics. In section 3, the dynamic feedback control approach is presented based on the nonlinear model of the vehicle. Feedback linearization-based controllers are determined in a disturbance-free case as well as in the presence of unknown but bounded aerodynamic disturbances and measurement noises. Section 4 focuses on the fixed-time differentiator design in the two cases. Then, numerical simulations are carried out to illustrate the efficiency of the whole observer-estimator-controller model.

a finite time $T\left(x_{0}\right)$. The settling time $T\left(x_{0}\right)$ is a finite value variant with initial conditions.

${ }^{2} \mathrm{~A}$ system is called fixed-time stable means that the system reaches in the steady state and remains there in a fixed time $T$. The settling time $T$ is a uniform value for a set of admissible initial states within the attraction domain. 


\section{Quadrotor dynamics}

The quadrotor is a nonlinear under-actuated dynamic system with four inputs and six degrees of freedom. It is composed of four individual rotors and a rigid cross airframe. Different motions are accomplished by changing the angular speed of propellers, which further change the thrust and moments.

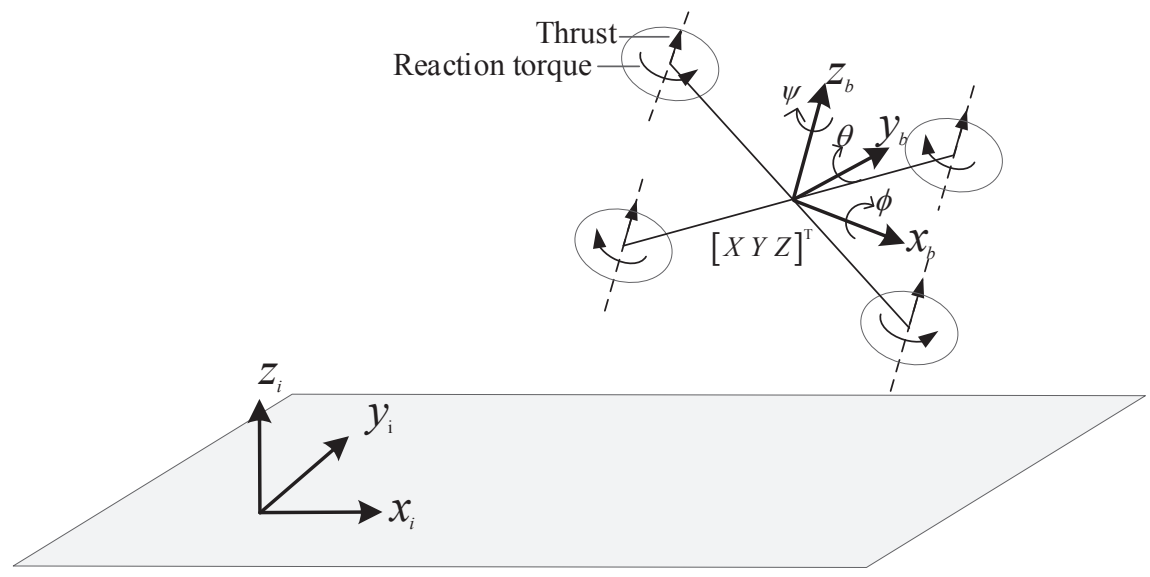

Figure 2. Quadcopter's dynamic scheme

In order to derive kinematic and dynamic equations of the quadrotor, two frames of reference should be introduced first. The inertial frame is associated with the ground, as is shown in Fig. 2, with gravity pointing in the negative $\boldsymbol{z}$ direction ${ }^{3}$. The vector $P=\left[\begin{array}{lll}X & Y & Z\end{array}\right]^{T}$ denotes the position of the center of mass of the vehicle. And the vector $V=\left[\begin{array}{lll}V_{x} & V_{y} & V_{z}\end{array}\right]^{T}$ denotes the linear velocity of the vehicle. The body fixed frame is associated with the vehicle and defined by the orientation of the quadrotor.

Here Euler angles $\Theta=\left[\begin{array}{lll}\psi & \theta & \phi\end{array}\right]^{T}$ are used to model the attitude of the quadrotor. These angles are denoted by yaw angle $\psi(-\pi \leq \psi<\pi)$, pitch angle $\theta\left(-\frac{\pi}{2}<\theta<\frac{\pi}{2}\right)$, and roll angle $\phi\left(-\frac{\pi}{2}<\theta<\frac{\pi}{2}\right)$ respectively. And the vector $\omega=\left[\begin{array}{lll}p & q & r\end{array}\right]^{T}$ denotes the angular velocity, which is derivatives of Euler angles with respect to time, expressed in the body frame. The rotation order from the inertial frame to the body frame is the yaw angle $\psi$ about the $\boldsymbol{z}$, then the pitch angle $\theta$ about $\boldsymbol{y}$, and the roll angle $\phi$ about $\boldsymbol{x}$. Thus the rotation matrix is as follows ${ }^{4}$ :

$$
R=\left[\begin{array}{ccc}
C \psi C \theta & C \psi S \theta S \phi-S \psi C \psi & C \psi S \theta C \phi+S \phi S \psi \\
C \theta S \psi & S \psi S \theta S \phi+C \psi C \phi & S \psi S \theta C \phi-C \psi S \phi \\
-S \theta & C \theta S \phi & C \phi C \theta
\end{array}\right]
$$

\footnotetext{
${ }^{3}$ In this paper, $\boldsymbol{x}, \boldsymbol{y}, \boldsymbol{z}$ in lower-case letters denote the three directions associated with the earth coordinate system.

${ }^{4}$ In this paper, $S, C, T$ and $S_{e}$ denote respectively $\sin , \cos , \tan$ and $s e c$.
} 
$J$ denotes the inertia matrix:

$$
J=\left[\begin{array}{ccc}
I_{x} & 0 & 0 \\
0 & I_{y} & 0 \\
0 & 0 & I_{z}
\end{array}\right]
$$

The rigid body equations of motion are:

$$
\begin{aligned}
\dot{P} & =V \\
m \dot{V} & =\sum F_{\text {ext }} \\
\dot{\Theta} & =W \omega \\
J \dot{\omega} & =-\omega \times(J \omega)+\sum \tau_{\text {ext }}
\end{aligned}
$$

where

$$
W=\left[\begin{array}{ccc}
0 & S \phi S_{e} \theta & C \phi S_{e} \theta \\
0 & C \phi & -S \phi \\
1 & S \phi T \theta & C \phi T \theta
\end{array}\right]
$$

Let $m$ denote the mass of the quadrotor, $l$ is the characteristic distance of the vehicle, and $g$ is the gravity constant. $u=\left[\begin{array}{llll}u_{1} & u_{2} & u_{3} & u_{4}\end{array}\right]^{T}$ is control input, with $u_{1}=F_{1}+F_{2}+F_{3}+F_{4}, u_{2}=l\left(F_{4}-F_{2}\right), u_{3}=l\left(F_{3}-F_{1}\right), u_{4}=c\left(F_{1}-F_{2}+F_{3}-F_{4}\right)$, where $F_{1}, F_{2}, F_{3}, F_{4}$ are thrusts of each rotor, and $c$ is the force-to-moment scaling factor.

Let $F_{x}, F_{y}$ and $F_{z}$ denote the resulting aerodynamic forces acting on the vehicle in the direction $\boldsymbol{x}, \boldsymbol{y}, \boldsymbol{z}$ respectively. Similarly, $M_{p}, M_{q}$ and $M_{r}$ denote the resulting aerodynamic moments. $\sum F_{\text {ext }}$ and $\sum \tau_{\text {ext }}$ represent respectively the external forces and torques:

$$
\sum F_{\text {ext }}=\left[\begin{array}{c}
F_{x}-(C \psi S \theta C \phi+S \phi S \psi) u_{1} \\
F_{y}-(S \psi S \theta C \phi-C \psi S \phi) u_{1} \\
F_{z}+m g-C \phi C \theta u_{1}
\end{array}\right], \sum \tau_{e x t}=\left[\begin{array}{c}
M_{p}+u_{2} l \\
M_{q}+u_{3} l \\
M_{r}+u_{4} l
\end{array}\right]
$$

Remark 1. In fact, the aerodynamic forces and moments have not been taken into account in various kinds of literature, i.e., $F_{x}=F_{y}=F_{z}=M_{p}=M_{q}=M_{r}=0$. To be more realistic, this paper regards those forces and moments as unknown, but bounded disturbances, which will be analyzed in section 3.2.2.

\section{Feedback Linearization}

As mentioned in the introduction, the feedback linearization is a common method used in nonlinear system control of the following form:

$$
\begin{aligned}
& \dot{x}=f(x)+g(x) u \\
& y=h(x)
\end{aligned}
$$


where $x \in \mathbb{R}^{n}$ is the state vector, $y \in \mathbb{R}^{m}$ is the output vector and $u \in \mathbb{R}^{p}$ is the input vector. The objective of this approach is to design a suitable control input with $u=\alpha(x)+\beta(x) v$ that renders a linear input-output map between the new control input $v$ and the system output $y$.

The essence of the feedback linearization is a transformation from the original nonlinear system to an equivalent linear system by a change of variables and a proper control input. To ensure that the transformed system is equivalent to the original one, the transformation must be a diffeomorphism. That is, the transformation should not only be invertible, i.e., bijective, but both the transformation and its inverse are smooth enough so that the differentiability in the original coordinate system can be preserved in the new coordinate system.

\subsection{Reformulation of quadrotors' dynamics}

The quadrotors' dynamics have been given in section 2. As we can notice from the second equation of system (1), linear accelerations $\ddot{X}, \ddot{Y}$ and $\ddot{Z}$ are affected only by the control input $u_{1}$, which may make this control problem unsolvable. A practical approach is to introduce a chain of double integrators to delay the appearance of $u_{1}$ in derivatives of $X, Y$ and $Z$, which is the so-called dynamic feedback control law, as is proved in Mistler et al. (2001).

Introduce a chain of integrators to the dynamic system and define a new control input $\bar{u}$ instead of $u$ :

$$
\begin{aligned}
& u_{1}=\zeta ; \quad \dot{\zeta}=\eta ; \quad \dot{\eta}=\bar{u}_{1} \\
& u_{2}=\bar{u}_{2} ; \quad u_{3}=\bar{u}_{3} ; \quad u_{4}=\bar{u}_{4}
\end{aligned}
$$

The system state $x=\left[\begin{array}{llllllllllllll}X & Y & Z & \psi & \theta & \phi & V_{x} & V_{y} & V_{z} & \zeta & \eta & p & q & r\end{array}\right]^{T}$, and the output $y=\left[\begin{array}{llll}y_{1} & y_{2} & y_{3} & y_{4}\end{array}\right]^{T}=C x=\left[\begin{array}{llll}X & Y & Z & \psi\end{array}\right]^{T}$ with $C=\left[\begin{array}{ll}I_{4} & 0_{4 \times 8}\end{array}\right]$. Let $\nu=\left[\begin{array}{llll}\nu_{1} & \nu_{2} & \nu_{3} & \nu_{4}\end{array}\right]^{T}$ be a bounded measurement noise and $\bar{d}$ denote a bounded aerodynamic disturbance. The quadrotor dynamics can be reformulated as follows:

$$
\begin{aligned}
& \dot{x}=f(x)+\sum_{i=1}^{4} g_{i}(x) \bar{u}_{i}+\bar{d} \\
& y=h(x)=C x+\nu
\end{aligned}
$$


where

$$
\begin{aligned}
& f(x)=\left[\begin{array}{c}
V_{x} \\
V_{y} \\
V_{z} \\
\frac{S \phi}{C \theta} q+\frac{C \phi}{C \theta} r \\
C \phi q-S \phi r \\
p+q T \theta S \phi+r C \phi T \theta \\
-\frac{1}{m}(C \phi C \psi S \theta+S \phi S \psi) \zeta \\
-\frac{1}{m}(C \phi S \psi S \theta-S \phi C \psi) \zeta \\
g-\frac{1}{m}(C \theta C \phi) \zeta \\
\eta \\
0 \\
\frac{\left(I_{y}-I_{z}\right)}{I_{x}} q r \\
\frac{\left(I_{z}-I_{x}\right)}{I_{y}} p r \\
\frac{\left(I_{x}-I_{y}\right)}{I_{z}} p q
\end{array}\right] \\
& g_{1}(x)=\left[\begin{array}{llllllllllllll}
0 & 0 & 0 & 0 & 0 & 0 & 0 & 0 & 0 & 0 & 1 & 0 & 0 & 0
\end{array}\right]^{T}
\end{aligned}
$$

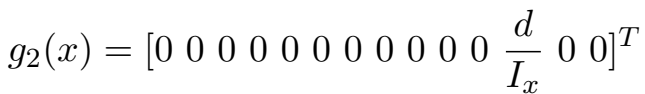

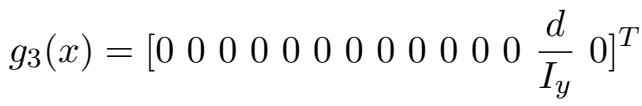

$$
\begin{aligned}
& g_{4}(x)=\left[\begin{array}{llllllllllllll}
0 & 0 & 0 & 0 & 0 & 0 & 0 & 0 & 0 & 0 & 1 & 0 & 0 & \frac{d}{I_{z}}
\end{array}\right]^{T}
\end{aligned}
$$

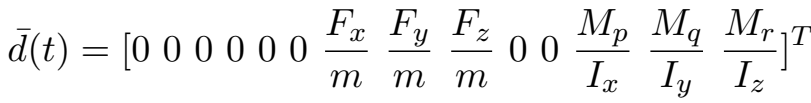

\subsection{Feedback linearization-based controller}

In this section, we first investigate the dynamics (2) of quadrotors without disturbance $\bar{d}$ ni measurement noise $\nu$, and then discuss the robustness for the obtained results when exogenous disturbance and measurement noise are involved.

\subsubsection{An ideal situation without disturbance ni noise}

In the disturbance- and noise-free case, the dynamic system (2) can be simplified as follows:

$$
\begin{aligned}
& \dot{x}=f(x)+\sum_{i=1}^{4} g_{i}(x) \bar{u}_{i} \\
& y=h(x)=C x
\end{aligned}
$$

For the given outputs of system (3), it is easy to verify that its relative degree ${ }^{5}$ $\left[\begin{array}{llll}r_{1} & r_{2} & r_{3} & r_{4}\end{array}\right]$ is given by:

$$
r_{1}=r_{2}=r_{3}=4 ; r_{4}=2
$$

\footnotetext{
${ }^{5}$ The relative degree of a system is the number of times of differentiations of the output $y$ before the control input $u$ appears explicitly. This is a notion that derives from Lie derivative.
} 
As we can notice, the dimension of system (3) is equal to 14, and its relative degrees satisfies

$$
\sum_{i=1}^{4} r_{i}=n=14
$$

According to Isidori (1989), the above equality implies that system (3) can be fully linearized without internal dynamics (i.e., the zero dynamics of the transformed system has zero dimension) by using the following diffeomorphism

$$
z=\Phi(x)=\left[h_{1}, L_{f} h_{1}, \cdots, L_{f}^{3} h_{1}, \cdots, h_{3}, L_{f} h_{3}, \cdots, L_{f}^{3} h_{3}, h_{4}, L_{f} h_{4}\right]^{T}
$$

yielding

$$
\begin{aligned}
& \dot{z}=A z+B(b(z)+\Delta(z) \bar{u}) \\
& y=C z
\end{aligned}
$$

where $b(z)$ and $\Delta(z)$ are determined by Lie derivative ${ }^{6}$ :

$$
\begin{aligned}
\Delta(z) & =\left[\begin{array}{ccc}
L_{g_{1}} L_{f}^{r_{1}-1} h_{1} & \cdots & L_{g_{4}} L_{f}^{r_{1}-1} h_{1} \\
\vdots & \ddots & \vdots \\
L_{g_{1}} L_{f}^{r_{4}-1} h_{4} & \cdots & L_{g_{4}} L_{f}^{r_{4}-1} h_{4}
\end{array}\right]_{\left.\right|_{x=\Phi^{-1}(z)}} \\
b(z) & =\left[\begin{array}{c}
L_{f}^{r_{1}} h_{1} \\
\vdots \\
L_{f}^{r_{4}} h_{4}
\end{array}\right]_{\left.\right|_{x=\Phi^{-1}(z)}}
\end{aligned}
$$

and

$$
A=\left[\begin{array}{cccc}
A_{1} & 0 & 0 & 0 \\
0 & A_{1} & 0 & 0 \\
0 & 0 & A_{1} & 0 \\
0 & 0 & 0 & A_{2}
\end{array}\right] \quad B=\left[\begin{array}{l}
B_{1} \\
B_{2} \\
B_{3} \\
B_{4}
\end{array}\right] \quad C=\left[\begin{array}{cccc}
C_{1} & 0 & 0 & 0 \\
0 & C_{1} & 0 & 0 \\
0 & 0 & C_{1} & 0 \\
0 & 0 & 0 & C_{2}
\end{array}\right]
$$

with

$$
\begin{array}{rlrl}
A_{1} & =\left[\begin{array}{llll}
0 & 1 & 0 & 0 \\
0 & 0 & 1 & 0 \\
0 & 0 & 0 & 1 \\
0 & 0 & 0 & 0
\end{array}\right] A_{2}=\left[\begin{array}{ll}
0 & 1 \\
0 & 0
\end{array}\right] \\
B_{1}=\left[\begin{array}{llll}
0 & 0 & 0 & 0 \\
0 & 0 & 0 & 0 \\
0 & 0 & 0 & 0 \\
1 & 0 & 0 & 0
\end{array}\right] \quad B_{2} & =\left[\begin{array}{llll}
0 & 0 & 0 & 0 \\
0 & 0 & 0 & 0 \\
0 & 0 & 0 & 0 \\
0 & 1 & 0 & 0
\end{array}\right] B_{3}=\left[\begin{array}{llll}
0 & 0 & 0 & 0 \\
0 & 0 & 0 & 0 \\
0 & 0 & 0 & 0 \\
0 & 0 & 1 & 0
\end{array}\right] \quad B_{4}=\left[\begin{array}{llll}
0 & 0 & 0 & 0 \\
0 & 0 & 0 & 1
\end{array}\right] \\
C_{1} & =\left[\begin{array}{llll}
1 & 0 & 0 & 0
\end{array}\right] & C_{2} & =\left[\begin{array}{ll}
1 & 0
\end{array}\right]
\end{array}
$$

${ }^{6}$ By definition, $L_{f} h(x)=\sum_{i=1}^{n} \frac{\partial h}{\partial x_{i}} f_{i}(x) ; L_{f}^{k} h(x)=L_{f}\left(L_{f}^{k-1} h(x)\right)$ 
It can be checked that the matrix $\Delta(z)$ defined in (6) is non-singular everywhere ${ }^{7}$ in the zone $\zeta \neq 0,-\frac{\pi}{2}<\phi<\frac{\pi}{2}$ and $-\frac{\pi}{2}<\theta<\frac{\pi}{2}$, which means that technically, there is no more limit for the pitch and roll angles.

The dynamic feedback approach transforms the original 12-dimensional system (1) into a 14-dimensional system (5) by introducing a chain of integrators. Thus, the goal is to design a proper controller to stabilize the outputs of the system (5).

Since $\Delta(z)$ is non-singular, by applying the following control law:

$$
\bar{u}=\alpha(z)+\beta(z) v
$$

where $\alpha(z)$ and $\beta(z)$ are given by

$$
\begin{aligned}
& \alpha(z)=-\Delta^{-1}(z) b(z) \\
& \beta(z)=\Delta^{-1}(z)
\end{aligned}
$$

then system (5) can be rewritten as

$$
\begin{aligned}
& \dot{z}=A z+B v \\
& y=C z
\end{aligned}
$$

for which different types of controllers can be easily designed.

\subsubsection{In the presence of external disturbance and measurement noise}

This subsection analyzes the disturbed dynamics of quadrotors.

By applying the same diffeomorphism (4), the system (2) can be transformed into

$$
\begin{aligned}
& \dot{z}=A z+B(b(z)+\Delta(z) \bar{u})+d(t) \\
& y=C z+\nu(t)
\end{aligned}
$$

where $A, B, C, b(z)$ and $\Delta(z)$ are the same as those defined in subsection 3.2.1, and

$$
d(t)=\left.\frac{\partial \Phi(x)}{\partial x}\right|_{\left.\right|_{x=\Phi^{-1}(z)}} \bar{d}(t)
$$

As $\bar{d}(t)$ and $\Phi(x)$ is a diffeomorphism, $d(t)$ is also bounded. In the bounded disturbance case, the objective is to design a proper controller such that the quadrotor can practically track the desired trajectory, i.e., converge into an acceptable neighborhood of the desired trajectory.

In the next section, a fixed-time differentiator will be designed for each subsystem in order to estimate $z$ of the disturbed system (9) with bounded errors and to reconstruct necessary information for the controller. The efficiency of this control strategy has been proved by a simulation comparison with the PID control strategy in the subsequent section.

\footnotetext{
${ }^{7}$ The non-singularity of $\Delta(x)$ has been proved in the paper of Mistler et al. (2001), we use directly the
} conclusion here. 


\section{Fixed-time differentiators with parameter tuning algorithm}

Real-time differentiation with convergence time constraints is a widely studied approach based on weighted homogeneity and implicit Lyapunov function, see Polyakov et al. (2015a). Due to the adjustability of the convergence time and the insensitivity to unknown inputs, the fixed-time differentiator is more attractive to be developed. Parameter tuning of the fixed-time differentiator, however, is still the toughest problem for implementation. In the paper of Lopez-Ramirez, Polyakov, Efimov, and Perruquetti (2016), an iteration algorithm with high efficiency has been proposed to tune the gain matrix of observation by using a Linear Matrix Inequality (LMI) optimization method. In the case of the quadrotor model, a simplified LMI-based parameter tuning algorithm can be developed for the fixed-time differentiators of each subsystem. The predefined fixed convergence time can be quickly settled via this algorithm.

\section{1. $\quad$ Fixed-time differentiators}

As presented in the previous section, the system has been transformed into four linear subsystems of $z_{1}, z_{2}, z_{3}$ and $z_{4}$, which correspond to the four channels $X, Y, Z$ and $\psi$ respectively. Each subsystem consists of one output signal and its derivatives. Thus, four fixed-time differentiators should be designed separately to observe the states of each subsystem. The fixed-time differentiator design and algorithms are identical for $X, Y, Z$, and are also similar for $\psi$, due to the similarity of these subsystems, as in (5). So in this section, both the theoretical method and computational approach are presented only for the first subsystem of $z_{1}$.

Consider the subsystem of $z_{1}=\left[\begin{array}{llll}X & \dot{X} & \ddot{X} & \dddot{X}\end{array}\right]^{T}$ :

$$
\begin{aligned}
& \dot{z}_{1}=A_{1} z_{1}+B_{1}(b(z)+\Delta(z) \bar{u})+d_{1}(t) \\
& y_{1}=C_{1} z_{1}+\nu_{1}
\end{aligned}
$$

where $A_{1}, B_{1}$ and $C_{1}$ are the first matrix blocks of $A, B, C$ defined in (5), and $d_{1}(t)$ is the first 4 rows of $d(t)$ defined in (10).

The observer of this subsystem is in this form:

$$
\dot{\hat{z}}_{1}=A_{1} \hat{z}_{1}+B_{1}(b(\hat{z})+\Delta(\hat{z}) \bar{u})+G\left(y_{1}-C_{1} \hat{z}_{1}\right)
$$

where

$$
G(\sigma)=\left(\frac{1}{2}\left(D_{\tilde{r}}\left(|\sigma|^{-1}\right)+D_{\tilde{r}}(|\sigma|)\right) L\right) \sigma
$$

with $L$ the gain matrix to be tuned, and $D_{\tilde{r}}$ the diagonal dilatation matrix in the form $(m=4$ for the subsystem (11)):

$$
D_{\tilde{r}}(\sigma)=\left[\begin{array}{cccc}
\sigma^{\tilde{r}_{1}} & 0 & \ldots & 0 \\
0 & \sigma^{\tilde{r}_{2}} & \ldots & 0 \\
\ldots & \ldots & \ldots & \ldots \\
0 & 0 & \ldots & \sigma^{\tilde{r}}
\end{array}\right]
$$

with $\tilde{r}=\left[\begin{array}{llll}\frac{\mu}{1+(m-1) \mu} & \frac{2 \mu}{1+(m-1) \mu} & \ldots & \frac{m \mu}{1+(m-1) \mu}\end{array}\right]^{T}$. 
The error equation for $e_{1}=\Phi\left(z_{1}-\hat{z}_{1}\right)$ has the form:

$$
\dot{e}_{1}=\left(A_{1}+\frac{1}{2}\left\{D_{\tilde{r}}\left(\left\|C_{1} e_{1}+\nu_{1}\right\|^{-1}\right)+D_{\tilde{r}}\left(\left\|C_{1} e_{1}+\nu_{1}\right\|\right)\right\} L \tilde{C}_{1}\right) e_{1}+\Delta \xi_{1}
$$

where $\Delta \xi_{1}=B_{1}(b(z)-b(\hat{z})+(\Delta(z)-\Delta(\hat{z})) \bar{u})+d_{1}(t)$ is bounded. It should be noted that in the disturbance free-case the error equation of the fixed-time differentiator (12) is a system homogeneous in the bi-limit for $\Delta \xi_{1}=0, \nu_{1}=0$ (see Andrieu, Praly, and Astolfi (2008) for more details about local homogeneity).

Let us denote

$$
\begin{aligned}
r_{i} & =(-1)^{i} \tilde{r}+\left[1+\frac{(-1)^{i+1} \mu}{1+(m-1) \mu}\right](1, \ldots, 1)^{T} \\
H_{i} & =\operatorname{diag}\left(\left(r_{i}\right)_{1},\left(r_{i}\right)_{2}, \ldots,\left(r_{i}\right)_{m}\right) \\
\bar{\Xi}_{i}(\lambda, \gamma) & =\frac{\lambda}{2}\left\{D_{\tilde{r}}\left(\frac{\gamma^{i-1}}{\lambda}\right)+D_{\tilde{r}}\left(\frac{\lambda}{\gamma^{i-2}}\right)-2 I_{m}\right\} \\
\lambda & >0, \gamma>0, i=1,2
\end{aligned}
$$

With $m=4, \tilde{r}=\left[\begin{array}{llll}\frac{\mu}{1+3 \mu} & \frac{2 \mu}{1+3 \mu} & \frac{3 \mu}{1+3 \mu} & \frac{4 \mu}{1+3 \mu}\end{array}\right]^{T}$, then $r_{i}$ and $H_{i}(i \in\{1,2\})$ can be determined

$$
\begin{aligned}
r_{1} & =\left(1+\frac{\mu}{1+3 \mu}\right)\left[\begin{array}{llll}
1 & 1 & 1 & 1
\end{array}\right]^{T}-\tilde{r} ; r_{2}=\left(1-\frac{\mu}{1+3 \mu}\right)\left[\begin{array}{cccc}
1 & 1 & 1 & 1
\end{array}\right]^{T}+\tilde{r} \\
H_{i} & =\operatorname{diag}\left(r_{i}\right)
\end{aligned}
$$

Theorem 4.1. (Lopez-Ramirez et al. (2016))

Let $\nu_{1}=0, \Delta \xi_{1}=0$ of the first subsystem in system (5) and for some $\mu \in(0,1)$, $\alpha>0$ the system of matrix inequalities

$$
\begin{array}{r}
P>0, \quad Z_{i}>0, \text { for } i=1,2 . \\
{\left[\begin{array}{rr}
P A_{1}+A_{1} P+\tilde{C}^{T} Y+Y^{T} \tilde{C}+\alpha\left(P+P H_{i}+H_{i} P\right) & P \\
P & -Z_{i}
\end{array}\right] \leq 0} \\
{\left[\begin{array}{cc}
\alpha I_{k} & Y \\
Y^{T} & P
\end{array}\right] \geq 0} \\
P H_{i}+H_{i} P>0, \quad P \geq \delta \tilde{C}^{T} \tilde{C} \tilde{C}^{T} \tilde{C}, \quad 0<\delta<1 \\
\bar{\Xi}_{i}(\lambda, \gamma) Z_{i} \bar{\Xi}_{i}(\lambda, \gamma) \leq P, \quad \forall \lambda \in\left(0, \delta^{-\frac{1}{2}}\right], \quad \forall \gamma \in(0,1]
\end{array}
$$

be feasible with $P, Z_{1}, Z_{2} \in \mathbb{R}^{n \times n}, Y \in \mathbb{R}^{n_{1} \times n}$, then the error equation with $L=Y P^{-1}$ is globally fixed-time stable with $T_{\max } \leq 2 \frac{1+(m-1) \mu}{\alpha \mu}$.

This theorem ensures the stability of the observer and provides a possibility to adjust convergence time independently of initial conditions. In particular, $\alpha$ is the parameter for tuning of $T_{\max }$. To avoid some unstable behavior of the closed-loop system during the convergence phase some output based (e.g. PI controller) can be utilized on the time interval $\left[0, T_{\max }\right]$. The proof of Theorem 4.1 is based on the weighted homogeneity and the implicit Lyapunov function method, as in Polyakov, Efimov, and Perruquetti (2015b). 
As mentioned LMIs should be checked for all $\lambda \in\left[0, \delta^{-\frac{1}{2}}\right]$, the inequality (16) is too complicated to implement in practice. In order to simplify this inequality, a proposition is taken as:

Proposition 4.2. (Lopez-Ramirez et al. (2016))

Let $0=\lambda_{0}<\lambda_{1}<\ldots<\lambda_{N_{1}}=\delta^{-\frac{1}{2}}$ and $0=\gamma_{0}<\gamma_{1}<\ldots<\gamma_{N_{2}}=1$ for some fixed $\delta \in(0,1)$. If the matrices $S_{i}, Z_{i}, R_{i}, M_{i}, U_{i} \in \mathbb{R}^{n \times n}$ and the number $\beta>0$ satisfy the following LMIs

$$
\begin{aligned}
& S_{i}>0, Z_{i}>0, R_{i}>0, M_{i}>0, U_{i}>0 \\
& S_{i} H_{\tilde{r}}+H_{\tilde{r}} S_{i}>0 \\
& {\left[\begin{array}{ccc}
2 Z_{i}-Z_{i} H_{\tilde{r}}-H_{\tilde{r}} Z_{i} & 2 Z_{i}+Z_{i} H_{\tilde{r}}-H_{\tilde{r}} Z_{i} & 2 Z_{i}-H_{\tilde{r}} Z_{i} \\
2 Z_{i}-Z_{i} H_{\tilde{r}}+H_{\tilde{r}} Z_{i} & 2 Z_{i}+Z_{i} H_{\tilde{r}}+H_{\tilde{r}} Z_{i}+S_{i} & 2 Z_{i}+H_{\tilde{r}} Z_{i} \\
2 Z_{i}-Z_{i} H_{\tilde{r}} & 2 Z_{i}+Z_{i} H_{\tilde{r}} & 2 Z_{i}+R_{i}
\end{array}\right] \geq 0} \\
& {\left[\begin{array}{cc}
Z_{i} H_{\tilde{r}}+H_{\tilde{r}} Z_{i}-\beta Z_{i} & H_{\tilde{r}} Z_{i}-\beta Z_{i} \\
Z_{i} H_{\tilde{r}}-\beta Z_{i} & M_{i}-\beta Z_{i}
\end{array}\right] \geq 0} \\
& {\left[\begin{array}{cc}
2 M_{i}+(-1)^{i}\left(H_{\tilde{r}} M_{i}+M_{i} H_{\tilde{r}}\right) & 2 M_{i}+(-1)^{i} H_{\tilde{r}} M_{i} \\
2 M_{i}+(-1)^{i} M_{i} H_{\tilde{r}} & U_{i}
\end{array}\right] \geq 0} \\
& \bar{\Xi}_{i}\left(\lambda_{j}, \gamma_{s}\right) Z_{i} \bar{\Xi}_{i}\left(\lambda_{j}, \gamma_{s}\right)+\left(\lambda_{j}-\lambda_{j-1}\right) R_{i}+\frac{\lambda_{j}-\lambda_{j-1}}{4} D_{\tilde{r}}\left(\frac{\lambda_{j}}{\gamma_{s}^{i-2}}\right) S_{i} D_{\tilde{r}}\left(\frac{\lambda_{j}}{\gamma_{s}^{i-2}}\right) \\
& +\frac{\gamma_{s}^{\beta}-\gamma_{s-1}^{\beta}}{\beta \gamma_{s}^{\beta}}\left(\bar{\Xi}_{i}\left(\lambda_{j}, 0\right) M_{i} \bar{\Xi}_{i}\left(\lambda_{j}, 0\right)+\left(\lambda_{j}-\lambda_{j-1}\right) U_{i}\right) \leq P \\
& i=1,2, j=1,2, \ldots, N_{1}, s=1,2, \ldots, N_{2}
\end{aligned}
$$

then the inequality (16) holds.

This proposition provides sufficient feasibility of the inequality (16), which allows developing an iteration parameter tuning algorithm with fixed $\delta$ and $\mu$. Based on the theorem and the proposition, a simple computational algorithm to tune the gain matrix $L$ for a quadrotor is developed in this paper, which largely reduces the computational complexity.

The basic idea of the algorithm is straightforward, which is based on the smoothness of the function $\Xi$ with respect to $\lambda$ : to execute the LMI optimization with a small size of grid constructed over $\lambda \in\left[0, \delta^{-\frac{1}{2}}\right]$ and $\gamma \in(0,1)$, and then to check the obtained solution with the tightest parametric matrix condition. It can be mathematically proved that when $\mu$ tends to be small enough, and $\alpha$ tends to be large enough, the optimization with the parametric conditions mentioned above is nearly feasible.

First, use a small number of $\lambda$ and $\gamma$ to execute the optimization with the LMI conditions $(14)-(16)$ and (18) - (22). As the inequality (17) is a tighter condition than the inequalities in the proposition 4.2, then the obtained results are required to be examined by the inequality (17) with a more compact grid of $\lambda$ and $\gamma$. If the inequality (17) is satisfied, then the obtained matrix $L$ is applicable for the fixed-time differentiators. If not, it is necessary to re-execute the first step with a larger size grid of $\lambda$ and $\gamma$. By means of this algorithm, the computation complexity can be largely reduced. And the desired convergence time can also be clearly settled by adjusting the two parameters $\alpha$ and $\mu$. 


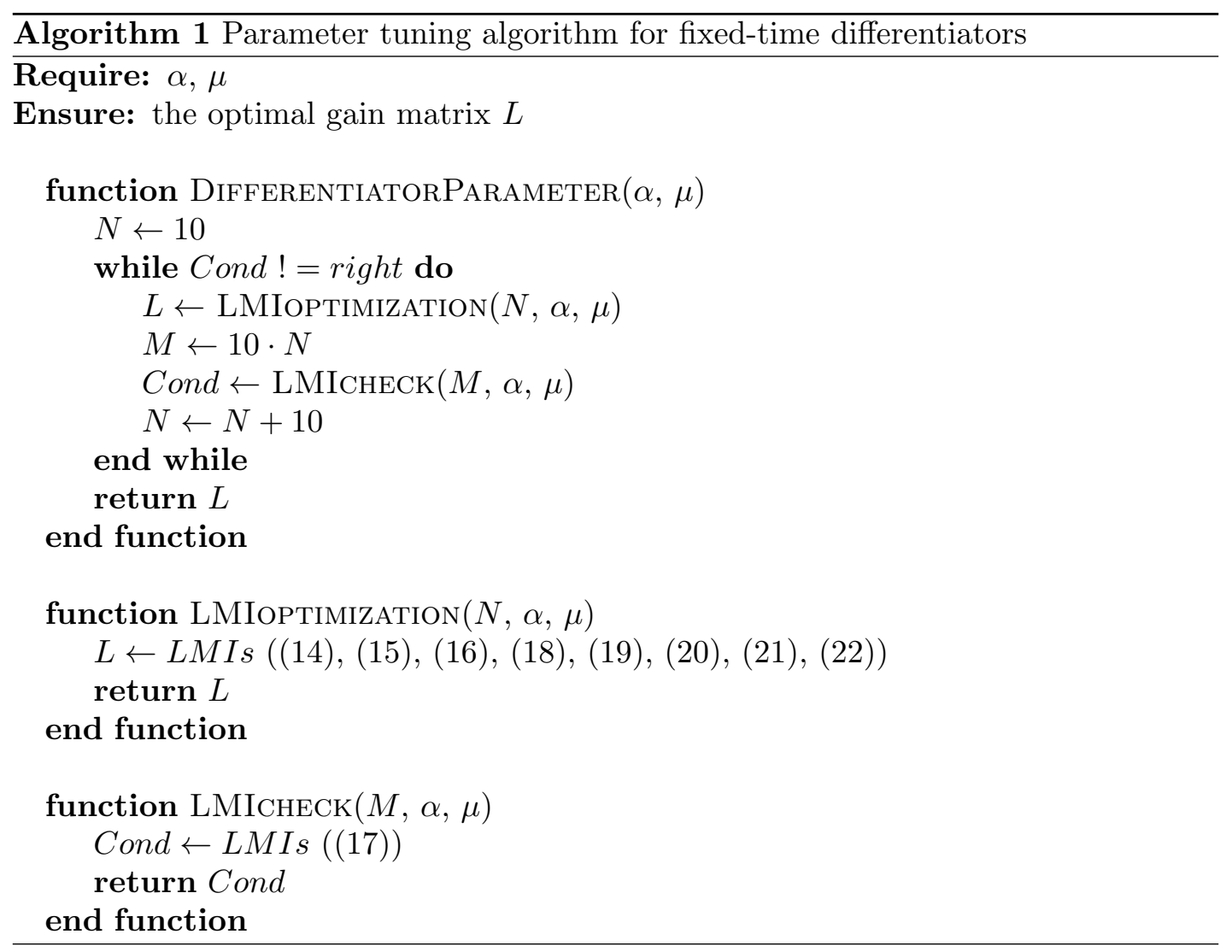

As the fixed-time differentiator designs for $X, Y$ and $Z$ are identical, the gain matrix $L$ is also the same for these three subsystems. In terms of the $\psi$ estimation, another two-dimensional gain matrix should be computed with the same algorithm.

Corollary 4.3. (Lopez-Ramirez, Polyakov, Efimov, and Perruquetti (2018))

Let conditions of Theorem 4.1 hold, but $\nu_{1} \neq 0$ and $\Delta \xi_{1} \neq 0$. Then the observer error dynamics is input-to-state stable with respect to $\Delta \xi_{1}$ and $\nu_{1}$.

For more details about input-to-state stability (ISS), readers can refer to Sontag and Wang (1996). Robustness analysis of homogeneous (in the bi-limit) systems is presented in Andrieu et al. (2008). In our case, it implies that $\|z(t)-\hat{z}(t)\| \leq \gamma(c)$ for $t \geq T_{\max }$, where $c \leq \max \{\|\nu\|,\|\Delta \xi\|\}$ and $\gamma:[0,+\infty) \rightarrow[0,+\infty)$ is a continuous strictly monotone function such that $\gamma(0)=0, \gamma(s)>0$ if $s>0$.

\subsection{State reconstruction}

Fixed-time differentiators presented above work as an observer for the output signal $\left[\begin{array}{llll}X & Y & Z & \psi\end{array}\right]$ and its derivatives. However, the observed values do not involve all variables of the original system. In order to obtain the full state information, the missed variables $\theta, \phi, p, q$ and $r$ should be reconstructed from observed values and 
nonlinear dynamic system (2). So $\hat{\theta}$ and $\hat{\phi}$ can be deduced as follows:

$$
\begin{aligned}
& \hat{\phi}=\arcsin \left(\frac{-m(\ddot{\hat{X}} S \psi-\ddot{\hat{Y}} C \psi)}{\zeta}\right) \\
& \hat{\theta}=\frac{1}{C \hat{\phi}} \arcsin \left(\frac{-m(\ddot{\hat{X}} C \psi+\ddot{\hat{Y}} S \psi)}{\zeta}\right)
\end{aligned}
$$

From the third equation of system (1), we know that the variables $\left[\begin{array}{lll}\hat{p} & \hat{q} & \hat{r}\end{array}\right]$ can be determined from angular velocity via the transformation matrix $W$. Therefore, $\dot{\hat{\phi}}$ and $\dot{\hat{\theta}}$ should be deduced first.

$$
\begin{aligned}
& \dot{\hat{\theta}}=-\frac{1}{C \hat{\theta} C \hat{\phi}^{2} \zeta}\left\{m \dddot{\hat{X}}(S \hat{\phi} S \hat{\theta} S \psi+C \psi C \hat{\phi})+m \dddot{\hat{Y}}(C \hat{\phi} S \psi-S \hat{\phi} C \psi S \hat{\theta})+\dot{\hat{\psi}} \zeta C \hat{\phi} S \hat{\phi} C \hat{\theta}^{2}-\zeta S \hat{\theta}\right\} \\
& \dot{\hat{\phi}}=\frac{1}{\zeta C \hat{\phi}}\{-m \dddot{\hat{X}} S \psi+m \ddot{\hat{Y}} C \psi+\psi \zeta C \hat{\phi} S \hat{\theta}+\zeta S \hat{\phi}\}
\end{aligned}
$$

Then the variables $\left[\begin{array}{lll}\hat{p} & \hat{q} & \hat{r}\end{array}\right]$ can be calculated by the following formula:

$$
\left[\begin{array}{c}
\hat{p} \\
\hat{q} \\
\hat{r}
\end{array}\right]=\left[\begin{array}{ccc}
1 & T \hat{\theta} S \hat{\phi} & T \hat{\theta} C \hat{\phi} \\
0 & C \hat{\phi} & -S \hat{\phi} \\
0 & S \hat{\phi} S_{e} \hat{\theta} & C \hat{\phi} S_{e} \hat{\theta}
\end{array}\right]^{-1}\left[\begin{array}{c}
\dot{\hat{\phi}} \\
\dot{\hat{\theta}} \\
\dot{\hat{\psi}}
\end{array}\right]
$$

By means of the state reconstruction step, the full system states have been obtained based on the values estimated by the fixed-time differentiators. All necessary information acquired by the feedback linearization-based controller is available for the whole closed loop.

\subsection{Outer loop design}

As we have mentioned previously, an outer-loop strategy can be applied to the linear control system after the input-output feedback linearization.

Different types of control laws can be used for the outer loop of the system, whether linear or nonlinear controllers, such as the polynomial controller, fixed-time controller, etc.

Following the formula (7) let us define the control $u$ as

$$
u=\alpha(\hat{z})+\beta(\hat{z}) v
$$

where

$$
\begin{aligned}
& v_{1}=X_{d}^{(4)}-K_{4} \dddot{e}_{11}-K_{3} \ddot{e}_{11}-K_{2} \dot{e}_{11}-K_{1} e_{11} \\
& v_{2}=Y_{d}^{(4)}-K_{4} \dddot{e}_{12}-K_{3} \ddot{e}_{12}-K_{2} \dot{e}_{12}-K_{1} e_{12} \\
& v_{3}=Z_{d}^{(4)}-K_{4} \dddot{e}_{13}-K_{3} \ddot{e}_{13}-K_{2} \dot{e}_{13}-K_{1} e_{13} \\
& v_{4}=\ddot{\psi}_{d}-K_{6} \dot{e}_{2}-K_{5} e_{2}
\end{aligned}
$$


where $X_{d}, Y_{d}, Z_{d}$ and $\psi_{d}$ represent the desired reference signals, $e_{11}=\hat{X}-X_{d}$, $e_{12}=\hat{Y}-Y_{d}, e_{13}=\hat{Z}-Z_{d}, e_{2}=\hat{\psi}-\psi_{d}$ are the error signals, and $K_{i}$ with $i \in[1,6]$ are the coefficients to be chosen to assign suitable eigenvalues. If $\hat{z}=z$ then the closed-loop system has form (8). This means that the equation describing evolution of the tracking error is linear and globally asymptotically stable. Consequently, it is input-to-state stable with respect to additive bounded perturbations, due to state estimation error $\|z-\hat{z}\| \leq \gamma(c)$ and the unknown exogenous disturbance $\bar{d} \neq 0$. Therefore, the practical stability of the error equation can be proved in the case of noised measurement and exogenous disturbances. The detailed qualitative analysis of the tracking error goes out of the scope of this paper and considered as an important problem for future research.

The whole observer-estimator-controller closed-loop system is presented in Fig. 3. By introducing a chain of double integrators, the feedback linearization approach transforms the dynamic system into four linear and controllable subsystems which correspond to the four output signals $X, Y, Z$ and $\psi$. The original nonlinear system is transformed into a set of independent channels. A fixed-time differentiator has been designed for each channel to observe and to estimate the output signals and its derivatives. Based on the values estimated by the fixed-time differentiators, the full state information required by the controller can be then deduced mathematically. Finally, a linear or a nonlinear control law can be implemented to the outer loop to render the system closed. The application of the fixed-time differentiator allows to realize the separation principle in nonlinear system. Since it converges within a fixed time $T_{\max }$, the whole system state is known for $t \geq T_{\max }$. Therefore, the controller using the full state estimation of the system can be effectively applied for $t \geq T_{\max }$.

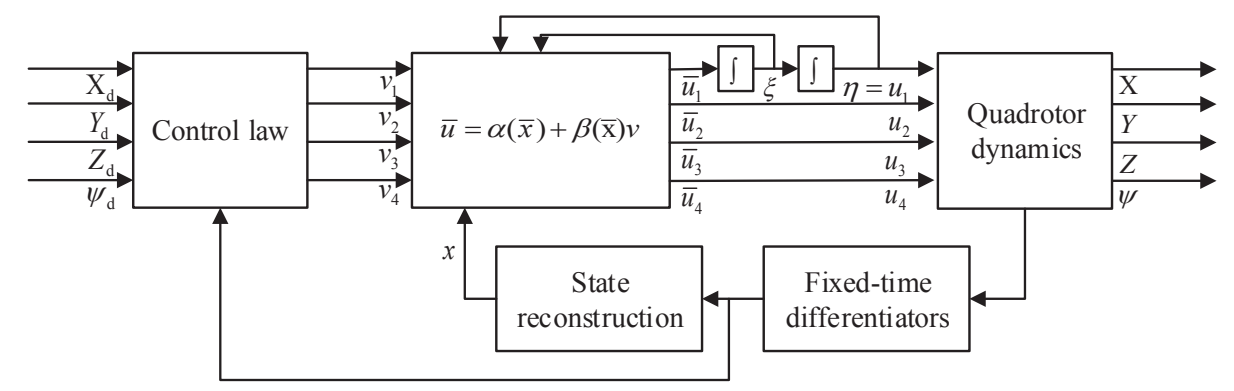

Figure 3. Observer-controller closed-loop system 


\section{Simulation study}

In this section, some simulations have been conducted to illustrate the theoretically established model. Parameters of the quadrotor model used here are:

$$
\begin{aligned}
& m=2 k g \quad d=0.1 m \quad g=9.81 \mathrm{~m} / \mathrm{s}^{2} \\
& I_{x}=I_{y}=I_{z}=1.2416 \mathrm{~N} \cdot \mathrm{m} / \mathrm{rad} / \mathrm{s}^{2}
\end{aligned}
$$

In order to verify the effectiveness of the fixed-time differentiators in the following study, the same trajectory has been imposed for $X, Y, Z$ and $\psi$ for all simulations, which is a continuous trajectory from 0 to 1 in 30 seconds.

\subsection{Control strategy comparison}

Before further presenting the whole observer-estimator-controller model, a simple comparison between the feedback linearization-based control strategy and the PID control strategy has been given.

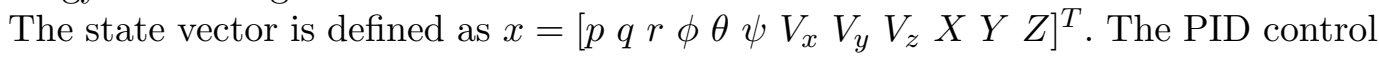
approach is applicable on a linear zone where the angles $\phi$ and $\theta$ are small enough $\left(<20^{\circ}\right)$. In this linear zone, the rotation matrix $R$, the matrix $W$, as well as the quadrotor dynamic functions (1) can be simplified. Thus, a linearized dynamic system has been obtained as follows:

$$
\begin{aligned}
& {\left[\begin{array}{l}
\dot{x}_{1} \\
\dot{x}_{2} \\
\dot{x}_{3}
\end{array}\right]=J^{-1}\left(\tau-\left[\begin{array}{ccc}
0 & -x_{3} & x_{2} \\
x_{3} & 0 & -x_{1} \\
-x_{2} & x_{1} & 0
\end{array}\right] J\left[\begin{array}{l}
x_{1} \\
x_{2} \\
x_{3}
\end{array}\right]\right)} \\
& {\left[\begin{array}{l}
\dot{x}_{4} \\
\dot{x}_{5} \\
\dot{x}_{6}
\end{array}\right]=\left[\begin{array}{ccc}
1 & \tan \left(x_{5}\right) \sin \left(x_{4}\right) & \tan \left(x_{5}\right) \cos \left(x_{4}\right) \\
0 & \cos \left(x_{4}\right) & -\sin \left(x_{4}\right) \\
0 & \sin \left(x_{4}\right) / \cos \left(x_{5}\right) & \cos \left(x_{4}\right) / \cos \left(x_{5}\right)
\end{array}\right]\left[\begin{array}{l}
x_{1} \\
x_{2} \\
x_{3}
\end{array}\right]} \\
& {\left[\begin{array}{l}
\dot{x}_{7} \\
\dot{x}_{8} \\
\dot{x}_{9}
\end{array}\right]=\left[\begin{array}{l}
0 \\
0 \\
g
\end{array}\right]-\frac{f}{m}\left[\begin{array}{c}
\cos \left(x_{6}\right) \sin \left(x_{5}\right) \cos \left(x_{4}\right)+\sin \left(x_{4}\right) \sin \left(x_{6}\right) \\
\sin \left(x_{6}\right) \sin \left(x_{5}\right) \cos \left(x_{4}\right)-\cos \left(x_{6}\right) \sin \left(x_{4}\right) \\
\dot{x}_{10} \\
\dot{x}_{11} \\
\dot{x}_{12}
\end{array}\right]=\left[\begin{array}{l}
x_{7} \\
x_{8} \\
x_{9}
\end{array}\right]}
\end{aligned}
$$

A hierarchical PID control approach, mentioned in the introduction, has been applied into this linearized system. Two PD controllers have been used in the position controller to obtain $\ddot{X}$ and $\ddot{Y}$, which further determine the desired angles $\phi_{d}$ and $\theta_{d}$ in the attitude planner. A PD controller has been used in the altitude controller for the channel of $Z$. Based on the angle errors $\Delta \phi, \Delta \theta$ and $\Delta \psi$, another PID controller has been used in the attitude controller to guarantee an exponential stability and to give the final commands to the dynamical system.

Simulation results of the PID control strategy and the feedback linearization control strategy have been given in the Fig.4. It can be noticed that the feedback linearization controller used for the trajectory tracking problem converges faster than the PID controller. And more importantly, the trajectory is more smooth which will be more 
compatible with the fixed-time differentiators.
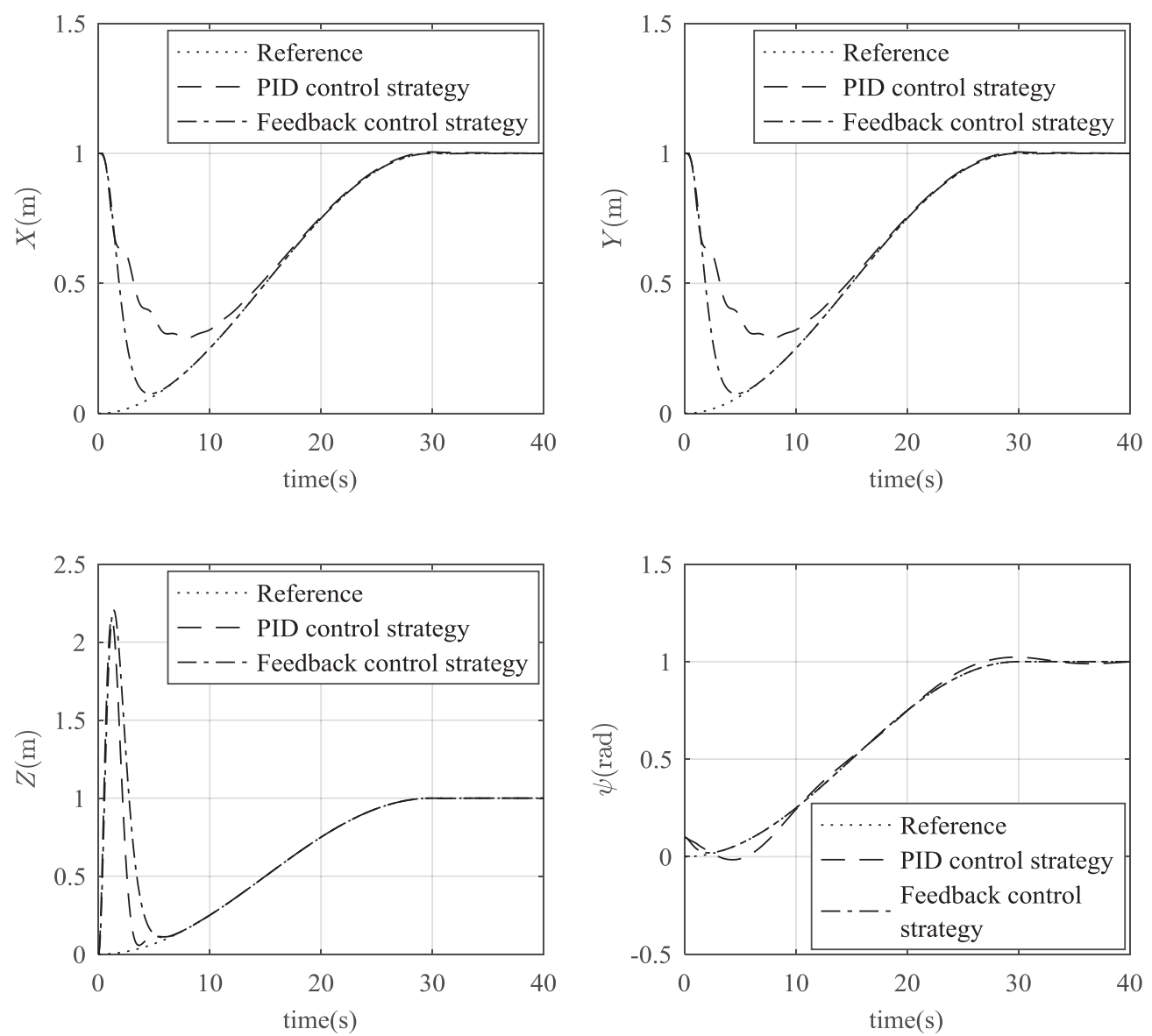

Figure 4. Control strategies' comparison

\subsection{Simulation study for the whole model}

Simulation studies have been conducted in this part to illustrate the performance of the whole observer-estimator-controller model.

Computational process has been presented in detail in the first part without considering the robustness of the differentiator. And simulation results with measurement noises and exogenous disturbance have also been presented to prove the performance of such a kind of design for quadrotor trajectory tracking problem.

\section{Disturbance- and noise-free case}

The gain matrix $L$ of the fixed-time differentiator is tuned first by setting $\mu=0.02$. According to the theorem, the maximum convergence time $T_{\max }$ can be predefined by 
choosing a suitable convergence rate $\alpha$. By setting $\alpha=40$, we can guarantee that the observer is stable within $T_{\max }=2.65 \mathrm{~s}$.
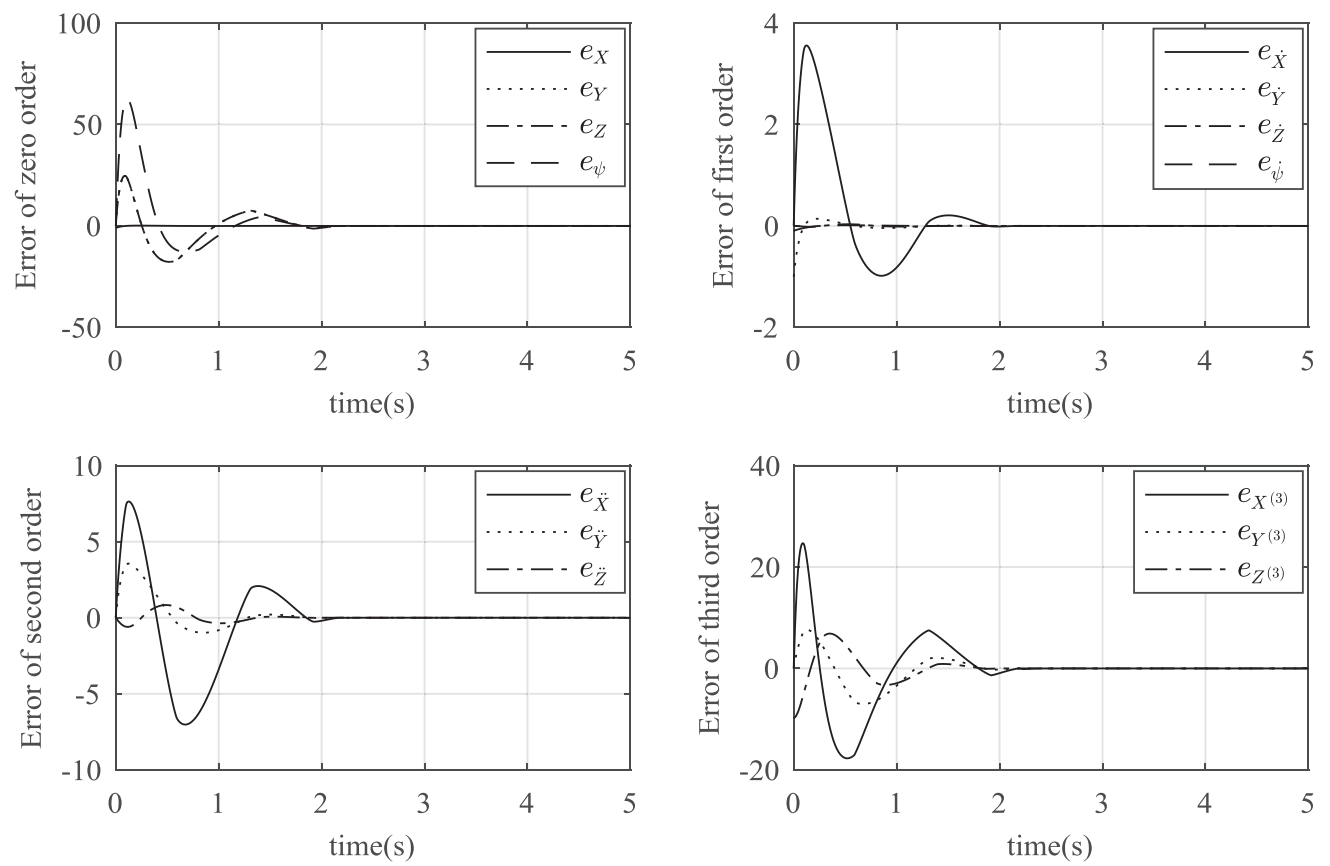

Figure 5. Estimation errors with respect to various initial differentiation errors

As differentiator designs are exactly the same for the estimation of $X, Y$ and $Z$, the compensation effect of initial differentiation errors can be proved by setting different initial values for these states. Fig. 5 depicts estimation errors of the fixed-time differentiators with respect to various initial differentiation errors (zoomed in $5 \mathrm{~s}$ ). Initial differentiation errors of $X$ and $Y$ have been settled to $1 \mathrm{~m}$, while initial differentiation error of $Z$ has been settled to $0 \mathrm{~m}$, and that of $\psi$ have been settled to $0.1 \mathrm{rad}$. It can be noticed that it is not necessary to give the differentiator the same initial values with the system initial conditions, because the fixed-time differentiator can provide a global stability independent of the initial conditions. And the system will stabilize after the predefined fixed convergence time $T_{\max }$. Furthermore, thanks to the LMI-based parameter tuning algorithm, we can easily obtain suitable parameters for differentiators and the convergence time $T_{\max }$ is also explicitly determined by the two parameters $\mu$ and $\alpha$, which largely reduces the complexity of the simulation.

The effectiveness of the observer-estimator-controller model is illustrated by the output signals $X, Y, Z$ and $\psi$, as shown in Fig. 6 . The dotted line is the predefined reference, while the full line represents the result of the closed loop. Different initial values have been assigned to different control channels in order to illustrate the performance of the proposed method. It can be concluded that the proposed observerestimator-controller scheme has satisfying efficiency in terms of accuracy and convergence speed with respect to different initial conditions. However, it appears that the fixed-time observer is highly sensitive to the sampling time and value assignment of $\mu$ and $\alpha$. The delicateness should be taken into account.

Moreover, the attitude of the quadrotor has also been examined. As it has been mentioned in the introduction, the feedback linearization is a linearization method 

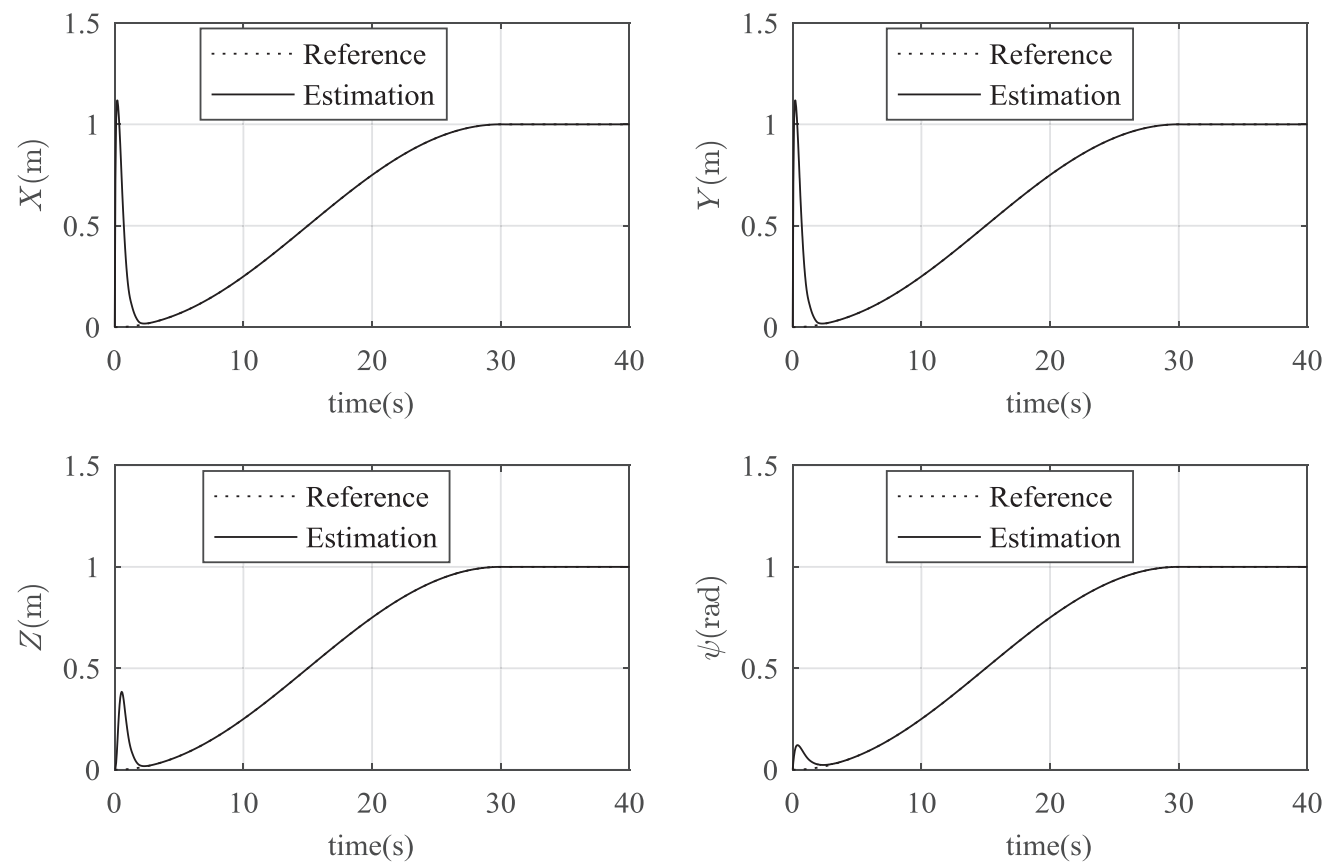

Figure 6. Output signals of the closed loop

from a global input-output point of view. Thus the classical limitations of small angles for pitch and roll angles have been removed. It is not necessary to bound the pitch and roll angles in a small interval. The pitch and roll angles should always stay in the zone of $-\frac{\pi}{2}<\phi<\frac{\pi}{2}$ and $-\frac{\pi}{2}<\theta<\frac{\pi}{2}$, which have been validated by the simulation result shown in Fig. 7 .

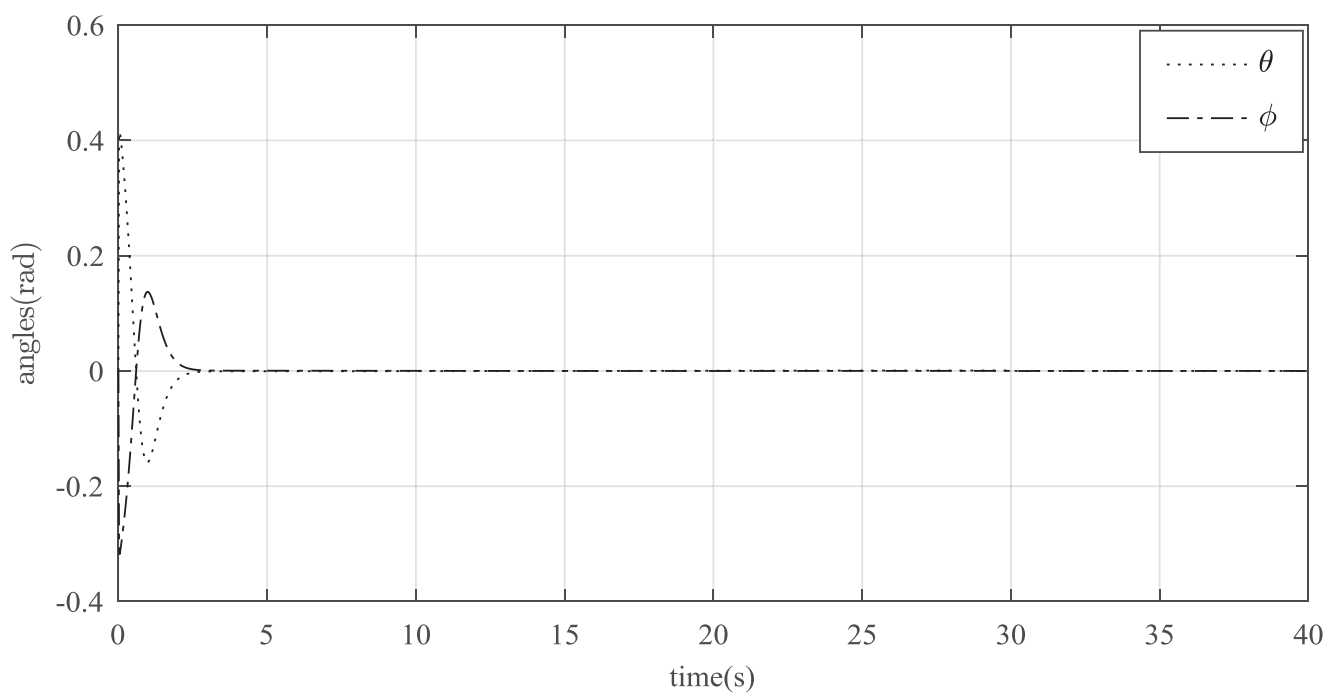

Figure 7. Pitch and roll angles 
With measurement noise and external disturbance

Considering input signals of the differentiator with random measurement noise of small amplitude (0.001), here the simulation results in the condition that initial observation errors equal zero:
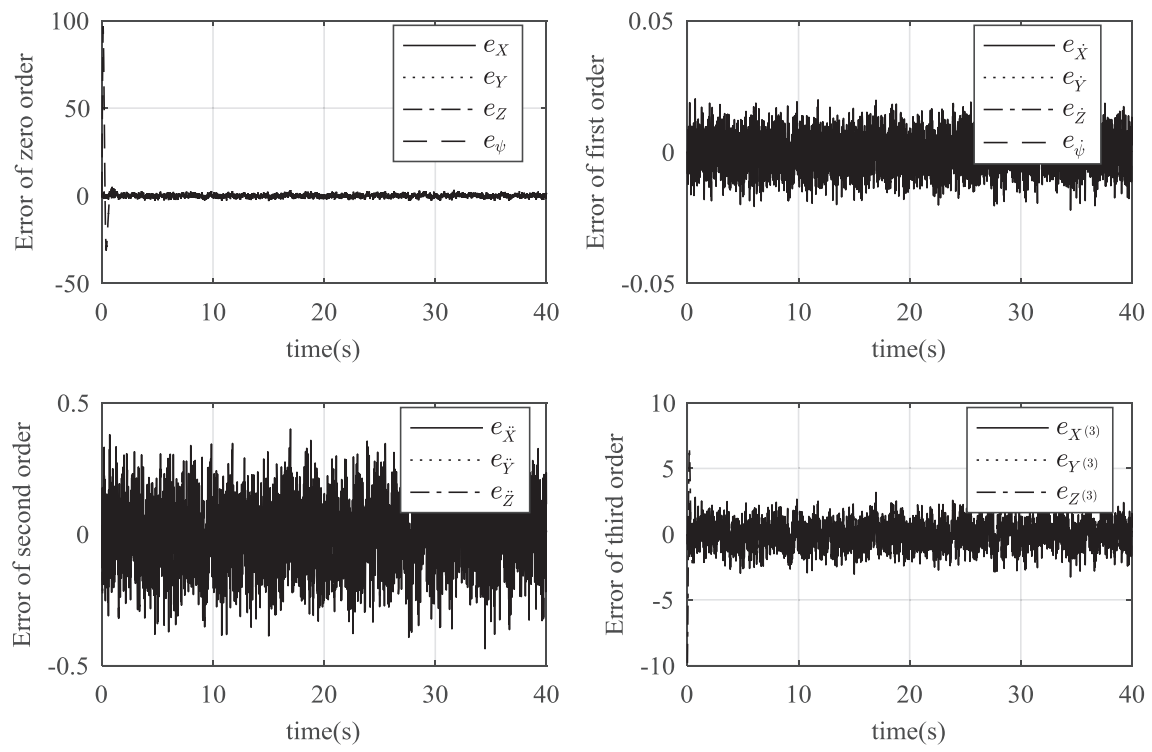

Figure 8. Errors with measurement noise
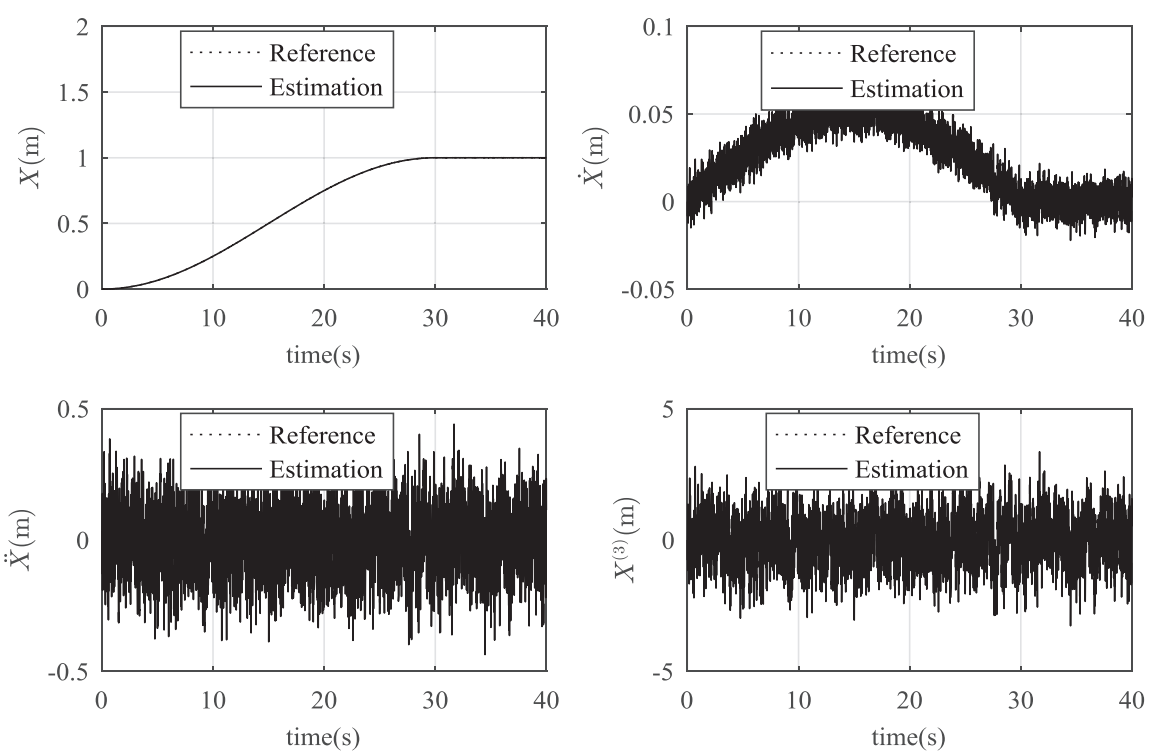

Figure 9. Simulation results of the channel $\mathrm{X}$ with measurement noise 

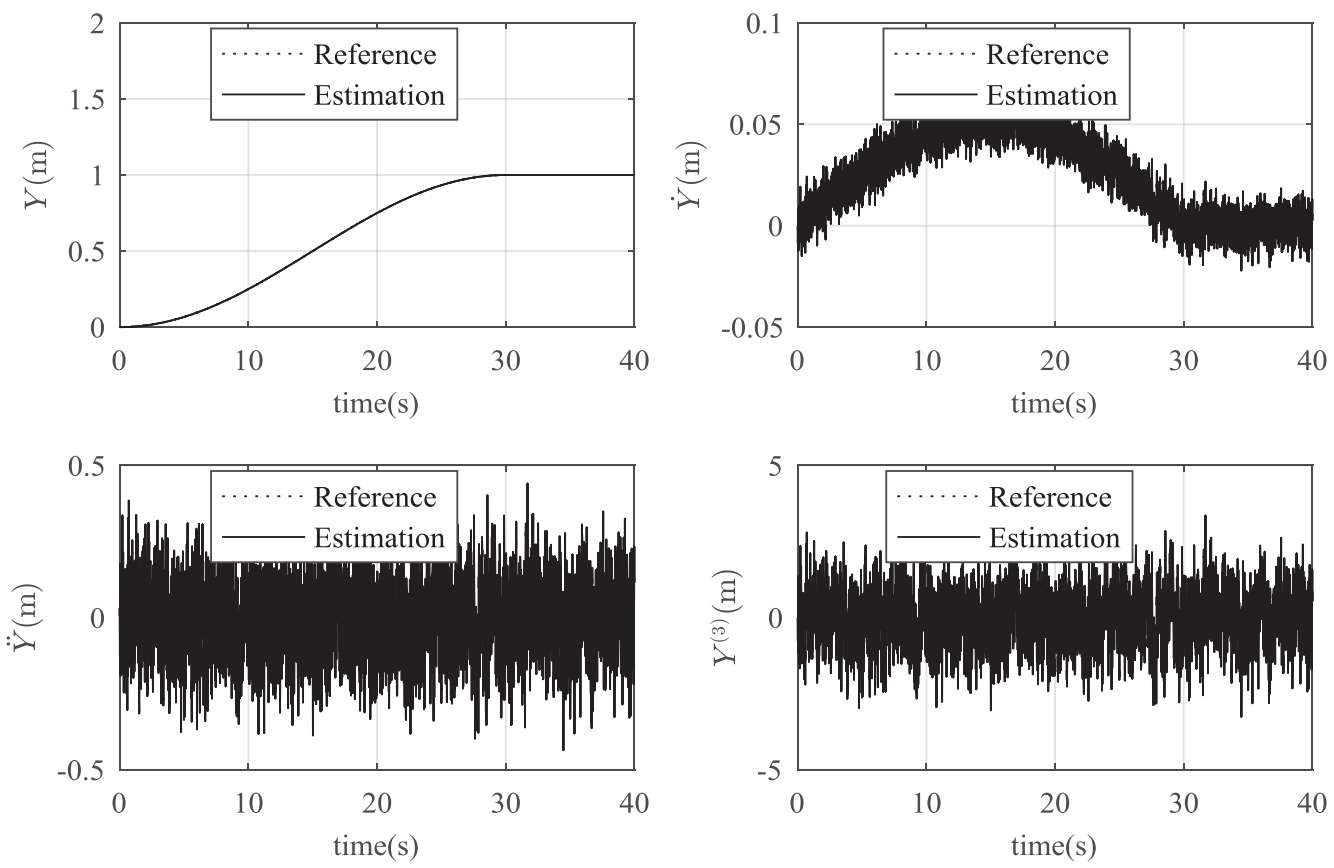

Figure 10. Simulation results of the channel $\mathrm{Y}$ with measurement noise
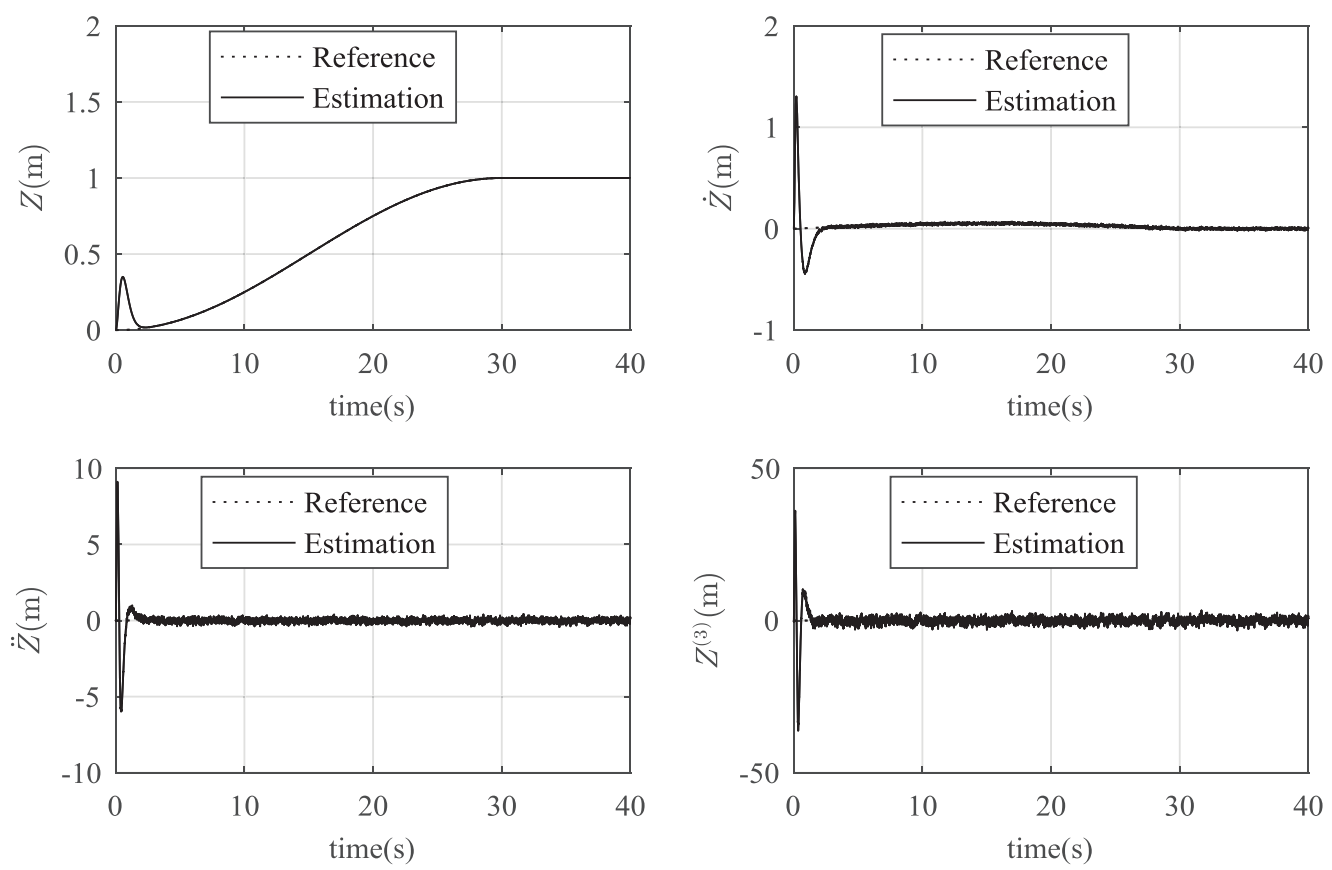

Figure 11. Simulation results of the channel $\mathrm{Z}$ with measurement noise 

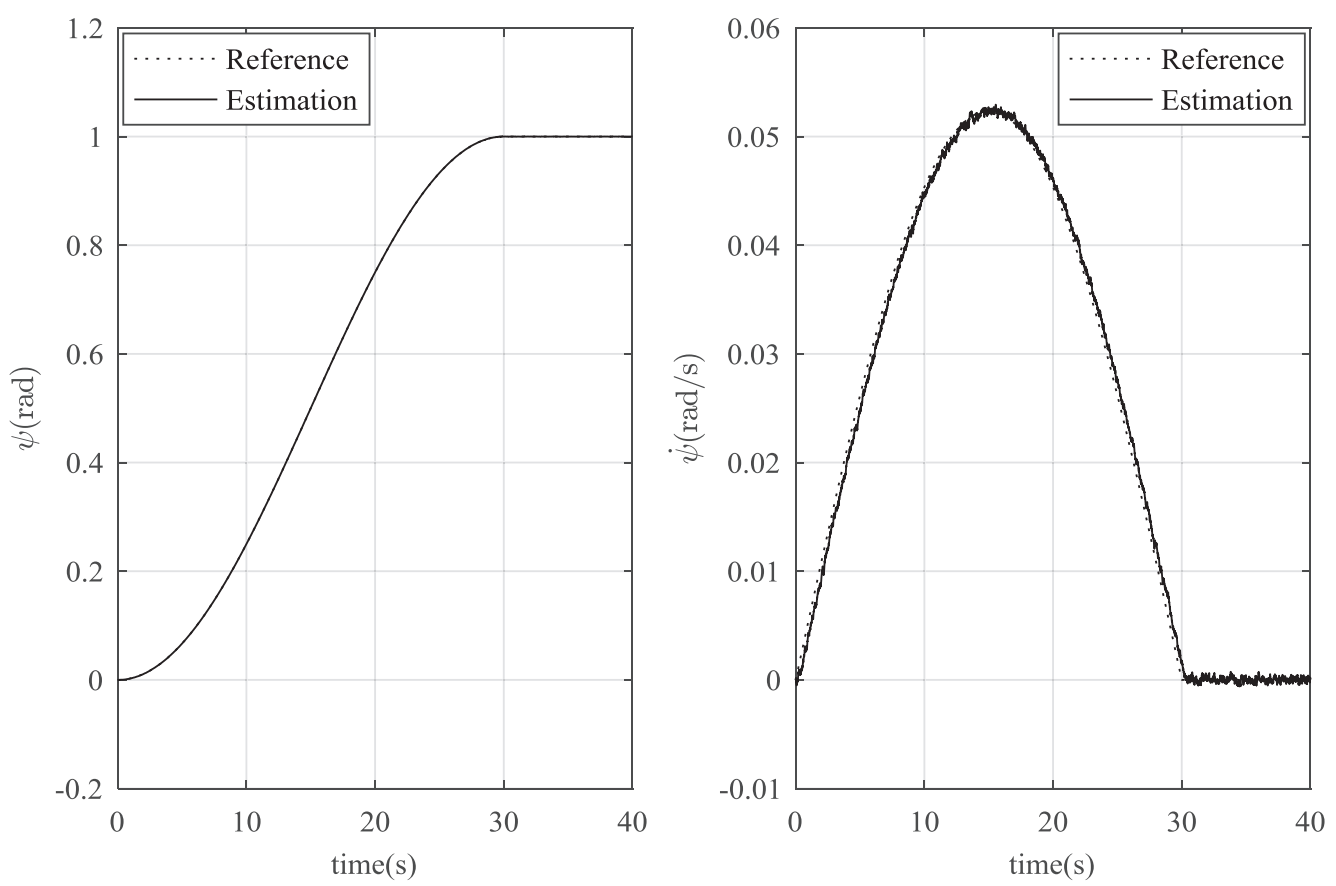

Figure 12. Simulation results of the channel $\psi$ with measurement noise

Then considering that an impulsive exogenous perturbation occurs to the system, which can be modeled as a short time constant perturbation with a very high amplitude, the simulation results are as follow:
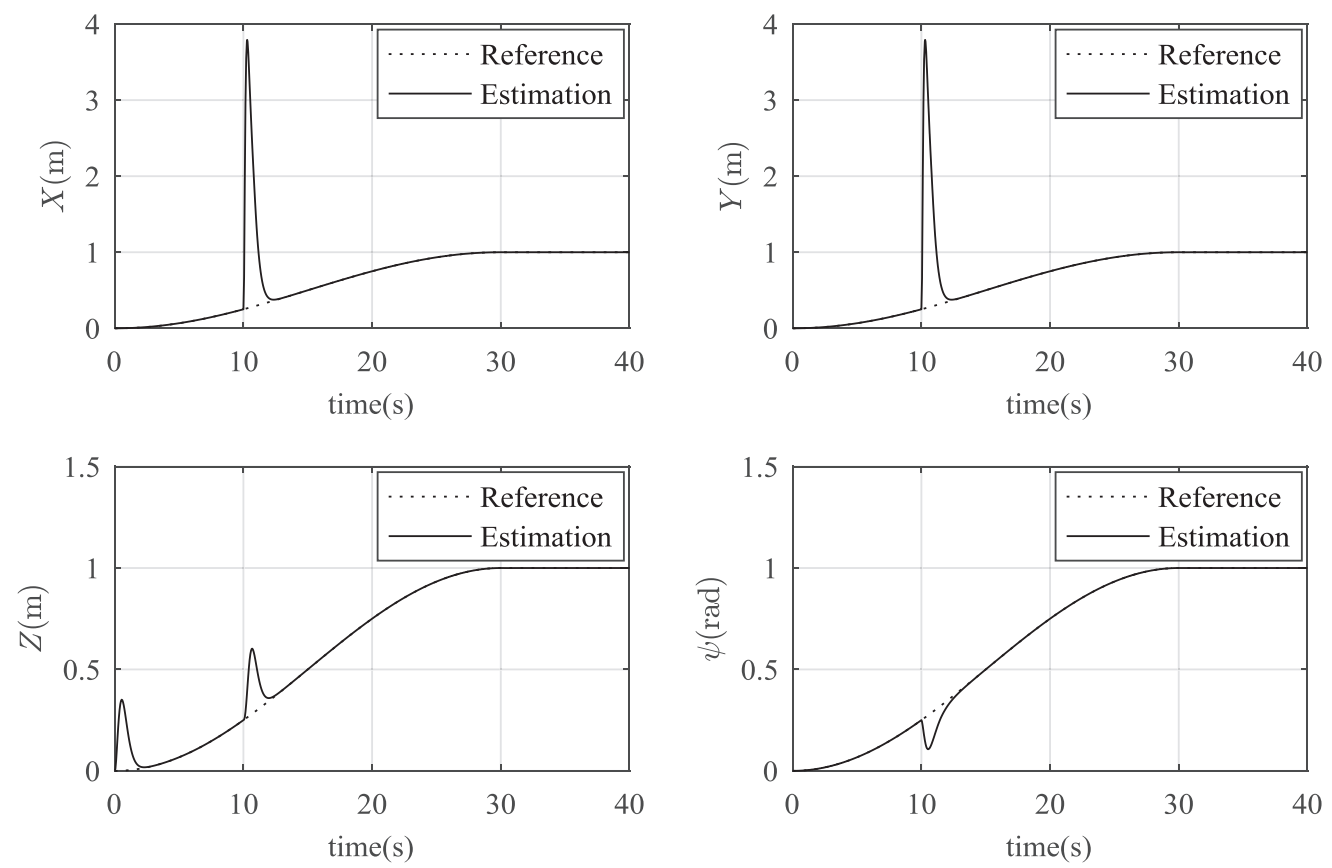

Figure 13. Simulation results in the presence of an impulsive perturbation 
In conclusion, the scheme proposed previously, a fixed-time differentiator running in parallel with a feedback linearization-based controller, allows the quadrotor to track a given trajectory. The fixed-time differentiator design guarantees a reliable estimation of the outputs' derivatives within a predefined convergence time with respect to different initial differentiation errors. Moreover, the LMI optimization-based parameter tuning algorithm provides the possibility to tune the settling time in an explicit form and reduces the computational complexity to obtain the parameters of the differentiator. The robustness of the fixed-time differentiator has been proved in the condition of measurement noise and an impulsive perturbation. The efficiency of the presented method has been illustrated through the simulation results.

\section{Conclusion}

This paper proposes an observer-estimator-controller scheme for trajectory tracking control of the quadrotor: a fixed-time differentiator running in parallel with a dynamic feedback linearization-based controller, where the gain matrix of the differentiator is tuned systematically by an LMI optimization-based algorithm.

The feedback linearization controller efficiently overcomes the nonlinearity and the decoupling problem of quadrotor dynamics, and compensates the limitations of small angles for the attitude of the quadrotor. The fixed-time differentiator works as an observer and an estimator in the closed-loop system, which provides exact estimated values regarding the requirement of full state information and the successive derivatives of the outputs. The LMI optimization-based parameter tuning algorithm provides a more accessible and practical approach to settle the convergence time explicitly and to obtain the gain matrix, largely reducing the computational complexity. As the convergence time is bounded by a fixed value independent of initial differentiation errors, the application of fixed-time differentiation and stabilization methods to quadrotors allows improving the delay problem and compensate the initial differatiation errors. Simulation results demonstrate the high performance of the proposed design for the quadrotor in autonomous flight. It can be observed that the stabilization of the whole system is bounded in a satisfying settling time with respect to different initial conditions. The robustness of the differentiator has been proved in the case of noise effects and impulsive disturbances. Moreover, the efficiency of the parameter tuning algorithm has also been proved.

For future studies, a qualitative analysis of tracking errors in the presence of additive bounded perturbations may be conducted. Also, a nonlinear controller, such as a fixedtime controller, is expected to replace the linear polynomial controller used in this paper.

\section{References}

Adigbli, P., Grand, C., Mouret, J. B., \& Doncieux, S. (2007). Nonlinear attitude and position control of a micro quadrotor using sliding mode and backstepping techniques. In EMAV conference and flight competition (p. 17-21). Toulouse, France.

Andrieu, V., Praly, L., \& Astolfi, A. (2008). Homogeneous approximation, recursive observer design, and output feedback. SIAM Journal on Control and Optimization, 47(4), 18141850 .

Angulo, M. T., Moreno, J. A., \& Fridman, L. $(2013,08)$. Robust exact uniformly convergent arbitrary order differentiator. Automatica, 49(8), 2489 - 2495. 
Austin, R. (2010). Unmanned aircraft systems: UAVs design, development and deployment. John Wiley \& Sons, Ltd.

Balas, C. (2007). Modeling and linear control of a quadrotor (Master dissertation). Cranfiel university.

Basin, M., Yu, P., \& Shtessel, Y. (2016). Finite and fixed setting time differentiators utilizing non-recursive higher order sliding mode control observer. In 14th international workshop on variable structure systems (p. 188-193). Nanjing, China.

Benallegue, A., Mokhtari, A., \& Fridman, L. (2007). Higher-order sliding-mode observer for a quadrotor uav. International Journal of Robust \&S Nonlinear Control, 18(4-5), 427-440.

Bouabdallah, S. (2006). Design and control of quadrotors with application to autonomous flying. Ph.D. dissertation, EPFL.

Bouabdallah, S., \& Siegwart, R. (2007). Full control of a quadrotor. In IEEE/RSJ international conference on intelligent robots and systems (p. 153-158). San Diego, CA, USA.

Cruz-Zavala, E., Moreno, J. A., \& Fridman, L. M. (2010, 11). Uniform robust exact differentiator. IEEE Transactions on Automatic Control, 56(11), 2727-2733.

Isidori, A. (1989). Nonlinear control systems. Springer-Verlag.

Khan, H., \& Kadri, M. B. (2014). Position control of quadrotor by embedded PID control with hardware in loop simulation. In 17th IEEE international multi-topic conference (INMIC) (p. 395-400). Karachi, Pakistan.

Levant, A. (1998, 03). Robust exact differentiation via sliding mode technique. Automatica, $34(3), 379-384$.

Levant, A. (2003). Higher-order sliding modes, differentiation and output-feedback control. International Journal of Control, 76(9/10), 924-941.

Lopez-Ramirez, F., Polyakov, A., Efimov, D., \& Perruquetti, W. (2016). Finite-time and fixed-time observers design via implicit lyapunov function. In European control conference (p. 2509-2514). Aalborg, Denmark.

Lopez-Ramirez, F., Polyakov, A., Efimov, D., \& Perruquetti, W. (2018, 01). Finite-time and fixed-time observers design: Implicit lyapunov function approach. Automatica, 87 .

Mahony, R., Kumar, V., \& Corke, P. (2012, 09). Multirotor aerial vehicles: Modeling, estimation, and control of quadrotor. IEEE Robotics 83 Automation Magazine, 19(3), 20-32.

Mistler, V., Benallegue, A., \& M'Sirdi, N. K. (2001). Exact linearization and noninteracting control of a 4 rotors helicopter via dynamic feedback. In IEEE international workshop on robot and human interactive communication (p. 586-593). Bordeaux, France.

Nijmeijer, H., \& van der Schaft, A. (1990). Nonlinear dynamical control systems. SpringerVerlag.

Polyakov, A. (2012). Nonlinear feedback design for fixed-time stabilization of linear control systems. IEEE Transactions on Automatic Control, 57(8), 2106-2110.

Polyakov, A., Efimov, D., \& Perruquetti, W. (2015a, 01). Finite-time and fixed-time stabilization: Implicit lyapunov function approach. Automatica, 51, 332-340.

Polyakov, A., Efimov, D., \& Perruquetti, W. (2015b, 01). Robust stabilization of MIMO systems in finite/fixed time. International Journal of Robust 83 Nonlinear Control, 26(1), 69-90.

Quan, Q. (2017). Introduction to multicopter design and control. Springer Singapore.

Rio, H., \& Teel, A. R. (2016). A hybrid observer for fixed-time state estimation of linear system. In IEEE 55th conference on decision and control (p. 5408-5413). Las Vegas, NV, USA.

Sontag, E., \& Wang, Y. (1996, 09). New characterizations of the input-to-state stability property. IEEE Transactions on Automatic Control, 41(9), 1283-1294. 